\title{
Pricing Analysis in Geo/Geo/1 Queueing System
}

\author{
Yan Ma and Zaiming Liu \\ School of Mathematics and Statistics, Central South University, Changsha, Hunan 410075, China \\ Correspondence should be addressed to Zaiming Liu; math_lzm@csu.edu.cn
}

Received 6 October 2014; Accepted 1 February 2015

Academic Editor: N. B. Naduvinamani

Copyright (C) 2015 Y. Ma and Z. Liu. This is an open access article distributed under the Creative Commons Attribution License, which permits unrestricted use, distribution, and reproduction in any medium, provided the original work is properly cited.

\begin{abstract}
This paper studies the equilibrium behavior of customers and optimal pricing strategies of servers in a Geo/Geo/1 queueing system. Two common pricing mechanisms are considered. The first one is called ex-post payment (EPP) scheme where the server collects tolls proportional to queue times, and the second one is called ex-ante payment (EAP) scheme where the server charges a flat fee for the total service. The server sets the toll price to maximize its own profit. It is found that, under a customer's choice equilibrium, the two toll mechanisms are equivalent from the economic point of view. Finally, we present several numerical experiments to investigate the effects of system parameters on the equilibrium customer joining rate and servers' profits.
\end{abstract}

\section{Introduction}

Analysis of discrete-time queues has always been of interest to practitioners because of its applications in digital communication and telecommunication networks. The study of discrete-time queues was first done by Meisling [1] and its importance has been noted in later decades. Then, Hunter [2] presented the mathematical techniques and applications in discrete-time models. Bruneel and Kim [3] investigated the discrete-time models for communication systems including ATM. Takagi [4] focused on the performance evaluation in discrete-time systems. Subsequently, more discrete-time queueing system studies have been conducted such as [5-8].

In this paper, we study the discrete-time $\mathrm{Geo} / \mathrm{Geo} / 1$ queue from an economic point of view. Naor [9] first studied equilibrium and socially optimal strategies in an $M / M / 1$ queue and, later, Naor's model was further extended by several authors, for example, Stidham [10] and Mendelson and Whang [11]. Recently, Ma et al. [12] and Wang et al. [13], respectively, analyzed the equilibrium customers' behavior in the $\mathrm{Geo} / \mathrm{Geo} / 1$ queue with multiple vacations and single working vacation. However, to the best of the authors' knowledge, there are relatively few studies on pricing problems in discrete-time queues.

We analyze the equilibrium strategies under two types of pricing structures. In the first structure, the server charges a fee that is proportional to the time a customer spends in the system, called ex-post payment (EPP) scheme. For example, in net bar, customers are charged by the hour when surfing the Internet. All using time including delays caused by network congestion in visiting websites is counted to be paid. Another common example is that a joining customer may be provided some kind of entertainment such as video games while he or she is waiting. Thus, it is reasonable to calculate the toll based on his or her sojourn time in the system. In the second structure, however, the server charges a fixed fee for the service, which means the server implements an exante payment (EAP) scheme. We analyze those two common pricing mechanisms. Initially, the two common toll policies (EPP and EAP) are investigated in batch-arrival queues by Sun et al. [14].

The paper is organized as follows. The model is formulated in Section 2. The customers' equilibrium joining and servers' profit maximization strategies under EPP and EAP schemes are given in Sections 3 and 4, respectively. In Section 5, we compare the two pricing structures numerically and examine the sensitivity of system parameters on the performance. Finally, Section 6 concludes with a brief summary.

\section{Model Description}

We consider a discrete-time Geo/Geo/1 queue in which the server sets a price to maximize his own benefit. Arriving customers do not know the real-time queue length and 
the decision to join or balk is irrevocable. The server follows a First-Come-First-Served (FCFS) service discipline.

Assume customer arrivals occur at the end of slot $t=$ $n^{-}, n=0,1, \ldots$. The interarrival times are independent and identically distributed (i.i.d.) random variables following a geometric distribution with rate $p(0<p<1)$. Note that $p$ is the potential arrival rate not the actual joining rate. We denote by $q$ the joining probability so that the actual rate of customers joining the queue is $p q$. Moreover, the service starting and service ending occur at slot division point $t=n, n=0,1, \ldots$. The service times are mutually independent and geometrically distributed with rate $\mu(0<$ $\mu<1)$. The interarrival times and service times are mutually independent. Thus, the expected time spent in the system by a joining customer $\omega=1 /(\mu-p q)$. For system stability, we assume $\mu>p q$.

We denote by $U$ the expected utility of a joining customer. After the completion of service, each customer receives a reward $R$. There exists a waiting cost of $C$ per time unit when the customer stays in the system. Customers are risk neutral. To make the model nontrivial, we assume the condition

$$
R>\frac{C}{\mu} .
$$

This condition ensures that the reward for service exceeds the expected cost for a customer who finds the system empty.

\section{EPP Scheme Model}

First, we consider the EPP scheme model, in which the server charges a price that is proportional to the time spent in the system. Suppose $K_{t}$ is the rate charged by the server and $P_{t}$ is the expected server profit per time unit.

Since the expected utility for a customer $U=R-K_{t} \omega-$ $C \omega$ and it equals zero in customers' equilibrium, we obtain $R=\left(K_{t}+C\right)(1 /(\mu-p q))$. Hence, the joining rate

$$
q=\frac{1}{p}\left(\mu-\frac{K_{t}+C}{R}\right) .
$$

Substituting (2) into $P_{t}=p q K_{t} \omega$, we get

$$
P_{t}=\frac{K_{t} R \mu}{C+K_{t}}-K_{t} .
$$

Since $\partial^{2} P_{t} / \partial K_{t}^{2}=-2 C R \mu /\left(C+K_{t}\right)^{3}<0$, which means $P_{t}$ is concave in $K_{t}$, we can maximize $P_{t}$ with respect to $K_{t}$ and obtain

$$
K_{t}=\sqrt{C R \mu}-C .
$$

Substituting (4) into (2), we have

$$
q_{t}=\frac{1}{p}\left(\mu-\sqrt{\frac{C \mu}{R}}\right) .
$$

If $q_{t} \leq 1, K_{t}$ in (4) is the optimal price. Substituting (4) into (3), we obtain the server's maximal profit:

$$
P_{t}=C+R \mu-2 \sqrt{C R \mu} .
$$

If $q_{t}>1$, the preferred joining rate $p q_{t}$ cannot be reached since $p$ poses an effective limitation. In this case, the server can increase its price without affecting the joining rate of the system, up to the point where $R=\left(K_{t}+C\right)(1 /(\mu-p))$. Hence, the optimal price and maximum profit for the server in this case are

$$
\begin{gathered}
K_{t}=(\mu-p) R-C, \\
P_{t}=p\left(R-\frac{C}{\mu-p}\right) .
\end{gathered}
$$

Based on the above analysis, we could give the following proposition.

Proposition 1. In EPP scheme model, denoting $q_{t}=(1 / p)(\mu-$ $\sqrt{C \mu / R})$, one has the following:

(1) if $q_{t} \leq 1$, there exists a unique equilibrium where customers join the queue with a probability $p q_{t}$ and $K_{t}=\sqrt{C R \mu}-C$ and $P_{t}=C+R \mu-2 \sqrt{C R \mu}$;

(2) if $q_{t}>1$, there exists a unique equilibrium where customers join the queue with a probability $p$ and $K_{t}=$ $(\mu-p) R-C$ and $P_{t}=p(R-C /(\mu-p))$.

\section{EAP Scheme Model}

Next, we consider the EAP scheme model, in which the server charges a flat price for service. Denote by $K_{f}$ the fixed price charged by the server and by $P_{f}$ the server expected profit per time unit.

Since the expected utility for a customer $U=R-K_{f}-C \omega$ and it equals zero in customers' equilibrium, we obtain $R=$ $K_{f}+C(1 /(\mu-p q))$. Hence, the joining rate

$$
q=\frac{1}{p}\left(\mu-\frac{C}{R-K_{f}}\right) .
$$

Substituting (9) into $P_{f}=p q K_{f}$, we have

$$
P_{f}=K_{f}\left(\mu+\frac{C}{K_{f}-R}\right) .
$$

Since $\partial^{2} P_{f} / \partial K_{f}^{2}=-2 C R /\left(R-K_{f}\right)^{3}<0$, which means $P_{f}$ is concave in $K_{f}$, we can maximize $P_{f}$ with respect to $K_{f}$ and obtain

$$
K_{f}=R-\sqrt{\frac{C R}{\mu}} .
$$

Substituting (11) into (9), we have

$$
q_{f}=\frac{1}{p}\left(\mu-\sqrt{\frac{C \mu}{R}}\right) .
$$

If $q_{f} \leq 1, K_{f}$ in (11) is the optimal price. Substituting (11) into (10), we obtain the server's maximal profit:

$$
P_{f}=C+R \mu-2 \sqrt{C R \mu} .
$$




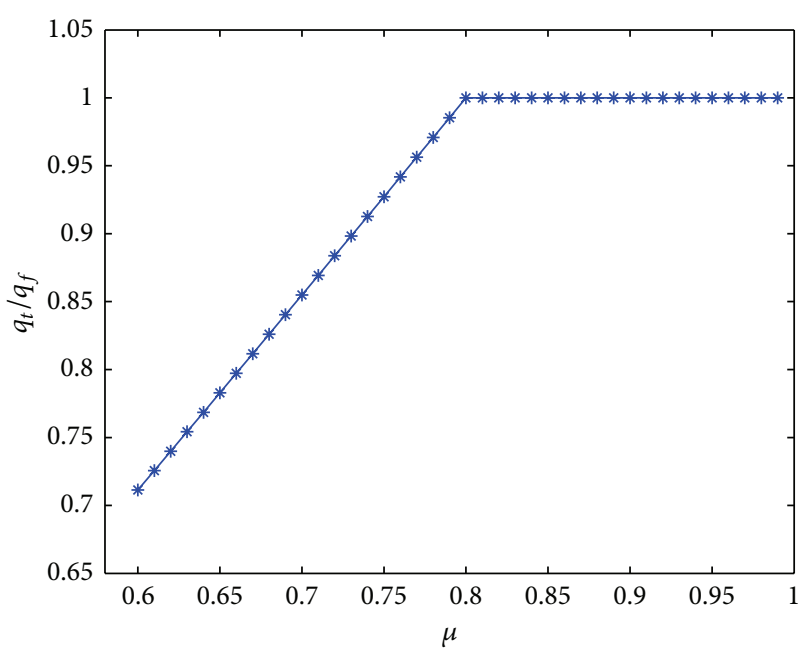

(a)

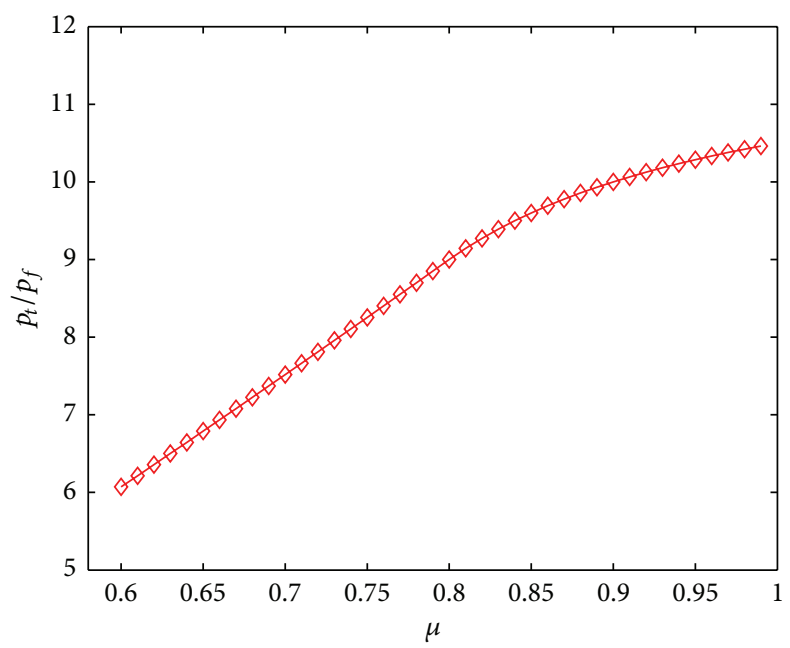

(b)

FIgURe 1: Sensitivity with respect to $\mu$ for $R=20, C=1$, and $p=0.6$.

If $q_{f}>1$, the preferred joining rate $p q_{f}$ can not be reached since $p$ poses an effective limitation. In this case, the server can increase its price without affecting the joining rate of the system, up to the point where $R=K_{f}+C(1 /(\mu-p))$. Hence, the optimal price and maximum profit for the server are

$$
\begin{gathered}
K_{f}=R-\frac{C}{\mu-p}, \\
P_{f}=p\left(R-\frac{C}{\mu-p}\right) .
\end{gathered}
$$

Based on the above analysis, we can give the following proposition.

Proposition 2. In EAP scheme model, letting $q_{f}=(1 / p)(\mu-$ $\sqrt{C \mu / R})$,

(1) if $q_{f} \leq 1$, there exists a unique equilibrium where customers join the queue with a probability $p q_{f}$ and $K_{f}=R-\sqrt{C R / \mu}$ and $P_{f}=C+R \mu-2 \sqrt{C R \mu}$;

(2) if $q_{f}>1$, there exists a unique equilibrium where customers join the queue with a probability $p$ and $K_{f}=$ $R-C /(\mu-p)$ and $P_{f}=p(R-C /(\mu-p))$.

\section{Analysis and Numerical Experiments}

Proposition 3. The two different charging mechanisms (EPP and EAP) do not affect the customer equilibrium joining rate and the server's maximum profits.

Proof. If the joining rate is not limited by $p$, that is, $q \leq 1$, from (5) and (12), after some calculations, we get $q_{t}-q_{f}=0$. In addition, from (6) and (13), we find $P_{t}=P_{f}$ which means the expected profit in EPP scheme is the same as that in EAP scheme.
Furthermore, if customers' joining rate is the maximum probability $p$, it is obvious that all customers enter the queue despite of different charging systems. From (8) and (15), we know the expected profits in EPP and EAP scheme models are the same.

Those results indicate that the customer equilibrium joining rate and the expected server profits are identical in the two different pricing schemes.

Based on the results obtained, we now present some numerical experiments. Here, we investigate the sensitivity of equilibrium joining rate $q\left(q_{t}\right.$ or $\left.q_{f}\right)$ for customers and expected server profits $P\left(P_{t}\right.$ or $\left.P_{f}\right)$ on system parameters.

From the subfigures (a) of Figures $1-3$, we can make the following observations. As the service rate and reward increase, the joining rate first goes up and then stays stable at the top point of 1 . This means that, within a certain range, more customers enter the system with a faster service or higher reward. All customers enter the queue when $\mu$ or $R$ exceeds a certain value. At the same time, the profit always grows when $\mu$ or $R$ increases. Hence, a high service rate or service reward for customers is also preferable for servers. Regarding the sensitivity of the arrival rate, it is easy to explain the system performance pattern. The system can attend to a certain number of customers and then beyond that point more customers joining will lead to congestion. Meanwhile, the server's profit stops increasing when no new customers enter the queue.

To give another example, we consider a scenario in cloud computing designed for business, also known as software as a service (SaaS). In SaaS, cloud providers install and operate application software and cloud users access the software from cloud clients to process complex data or optimize object functions, and so forth. SaaS is sometimes referred to as "on-demand software" and is usually priced on a pay-peruse basis or time basis. Basically, there is a single server and 


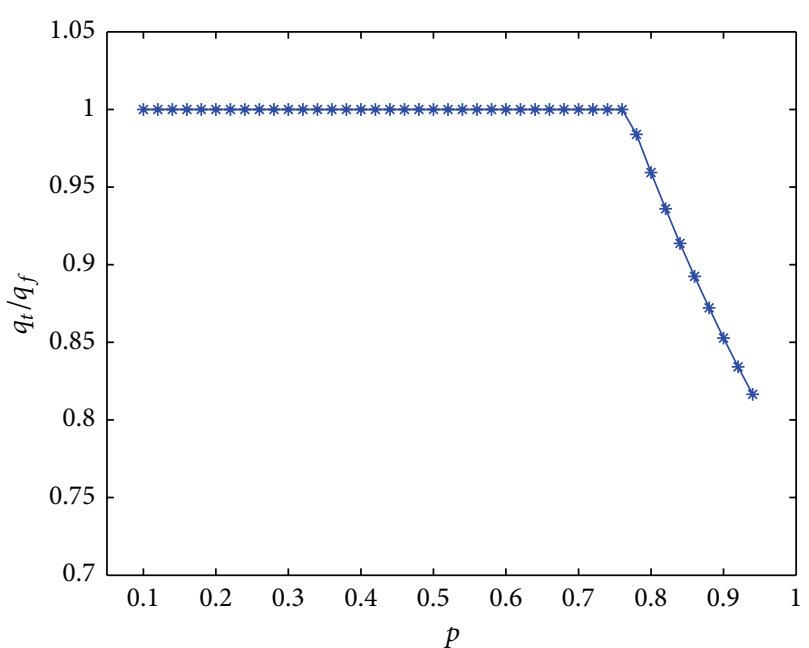

(a)

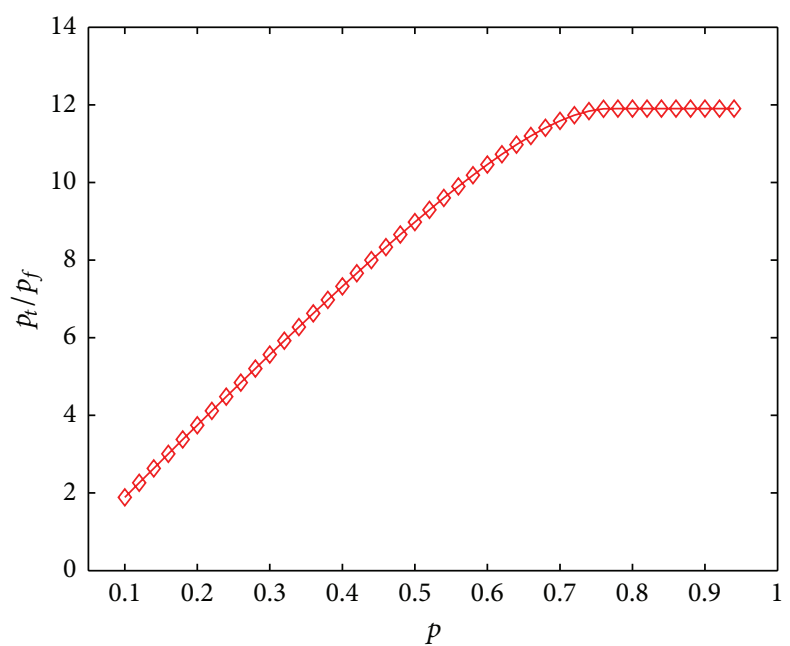

(b)

Figure 2: Sensitivity with respect to $p$ for $R=20, C=1$, and $\mu=0.99$.

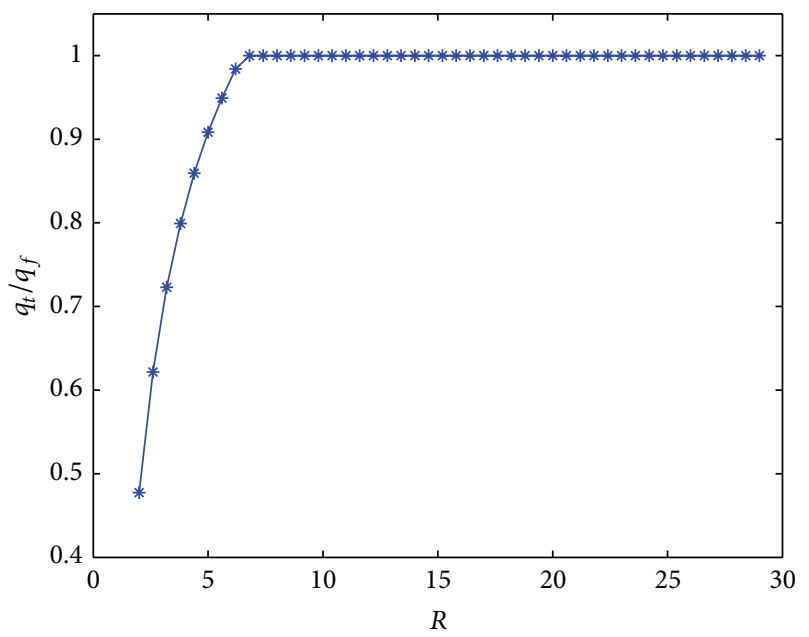

(a)

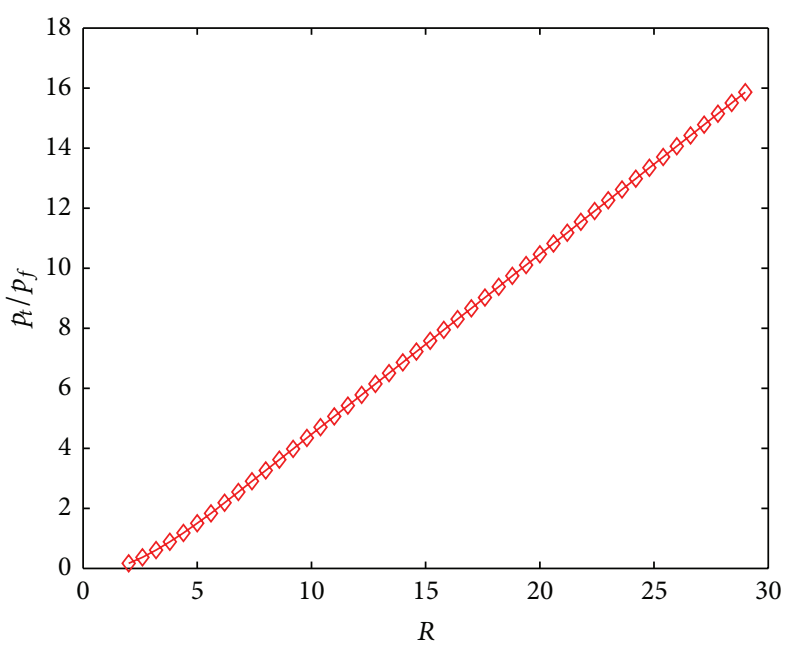

(b)

FIgURE 3: Sensitivity with respect to $R$ for $C=1, p=0.6$, and $\mu=0.99$.

interarrival times and service times are mutually independent and geometrically distributed.

Suppose the system parameters are given as $\mu=0.9, p=$ $0.6, R=20$, and $C=1$. If a flat fee is charged, we get $q_{t} \approx$ 1.15 , which belongs to the subcase (1) in Proposition 1. Thus, customer processing requests are better to follow the unique equilibrium entering rate 0.6 and the cloud provider could set the price per time unit as $K_{t}=5$ to obtain the maximal profit per time unit $P_{t}=10$.

As to concerning it in EAP scheme model, from Propositions 2 and 3, we know the processing request's equilibrium entering rate and the cloud provider's maximal profits are the same as those in EPP model. However, there is only one difference that the cloud provider could set a fixed price for per use as $K_{f}=16.67$ in EPP model.

\section{Conclusions}

We have studied customer equilibrium joining behavior and server's optimal pricing strategies. A server can choose between those two common pricing policies to maximize his profit. In EPP scheme, the server collects a fee that is proportional to the duration a customer is in queue, while in EAP scheme it charges a flat fee for the service. It is found that customers' equilibrium joining rates under the two different pricing schemes are the same. Moreover, the server's maximum benefits in the two pricing schemes are also identical.

These results could provide queueing managers with useful information for making the pricing decisions and instruct customers to take optimal strategies. A possible 
extension to this work can be to consider these pricing schemes in multiple server queueing systems.

\section{Conflict of Interests}

The authors declare that there is no conflict of interests regarding the publication of this paper.

\section{Acknowledgments}

This work was supported by the National Natural Science Foundation of China (no. 11271373) and Hunan Provincial Innovation Foundation for Postgraduate (no. CX2013B042). Yan Ma would like to thank China Scholarship Council for supporting her visit to Simon Fraser University.

\section{References}

[1] T. Meisling, "Discrete-time queuing theory," Operations Research, vol. 6, pp. 96-105, 1958.

[2] J. J. Hunter, Mathematical Techniques of Applied Probability, Academic Press, New York, NY, USA, 1983.

[3] H. Bruneel and B. G. Kim, Discrete-Time Models for Communication Systems Including ATM, Kluwer Academic Publishers, Boston, Mass, USA, 1993.

[4] H. Takagi, Queueing Analysis: A Foundation of Performance Evaluation, North-Holland, Amsterdam, The Netherlands, 1993.

[5] J. Kim, B. Kim, and J. Kang, "Discrete-time multiserver queue with impatient customers," Electronics Letters, vol. 49, no. 1, pp. 38-39, 2013.

[6] K. Kim and K. C. Chae, "Discrete-time queues with discretionary priorities," European Journal of Operational Research, vol. 200, no. 2, pp. 473-485, 2010.

[7] F. Zhang and Z. Zhu, "A discrete-time unreliable Geo/G/1 retrial queue with balking customers, second optional service, and general retrial times," Mathematical Problems in Engineering, vol. 2013, Article ID 832318, 12 pages, 2013.

[8] Z. Ma, Y. Guo, P. Wang, and Y. Hou, "The Geo/Geo/1+1 queueing system with negative customers," Mathematical Problems in Engineering, vol. 2013, Article ID 182497, 8 pages, 2013.

[9] P. Naor, "The regulation of queue size by levying tolls," Econometrica, vol. 37, no. 1, pp. 15-24, 1969.

[10] J. Stidham Jr., "Optimal control of admission to a queueing system," IEEE Transactions on Automatic Control, vol. 30, no. 8, pp. 705-713, 1985.

[11] H. Mendelson and S. Whang, "Optimal incentive-compatible priority pricing for the $M / M / 1$ queue," Operations Research, vol. 38, no. 5, pp. 870-883, 1990.

[12] Y. Ma, W.-Q. Liu, and J.-H. Li, "Equilibrium balking behavior in the $\mathrm{Geo} / \mathrm{Geo} / 1$ queueing system with multiple vacations," Applied Mathematical Modelling, vol. 37, no. 6, pp. 3861-3878, 2013.

[13] F. Wang, J. Wang, and F. Zhang, "Equilibrium customer strategies in the GEO/GEO/1 queue with single working vacation," Discrete Dynamics in Nature and Society, vol. 2014, Article ID 309489, 9 pages, 2014.

[14] W. Sun, S. Li, N. Tian, and H. Zhang, "Equilibrium analysis in batch-arrival queues with complementary services," Applied Mathematical Modelling, vol. 33, no. 1, pp. 224-241, 2009. 


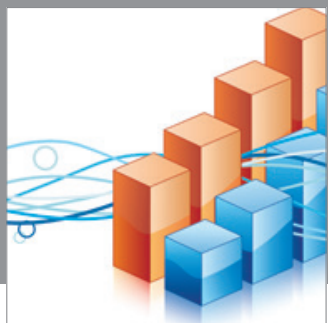

Advances in

Operations Research

mansans

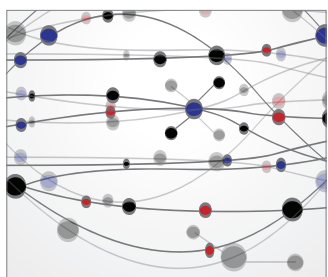

The Scientific World Journal
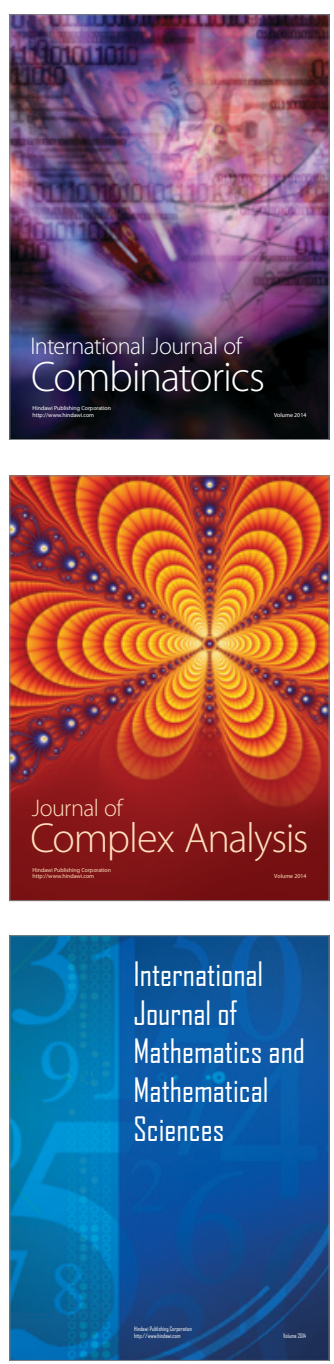
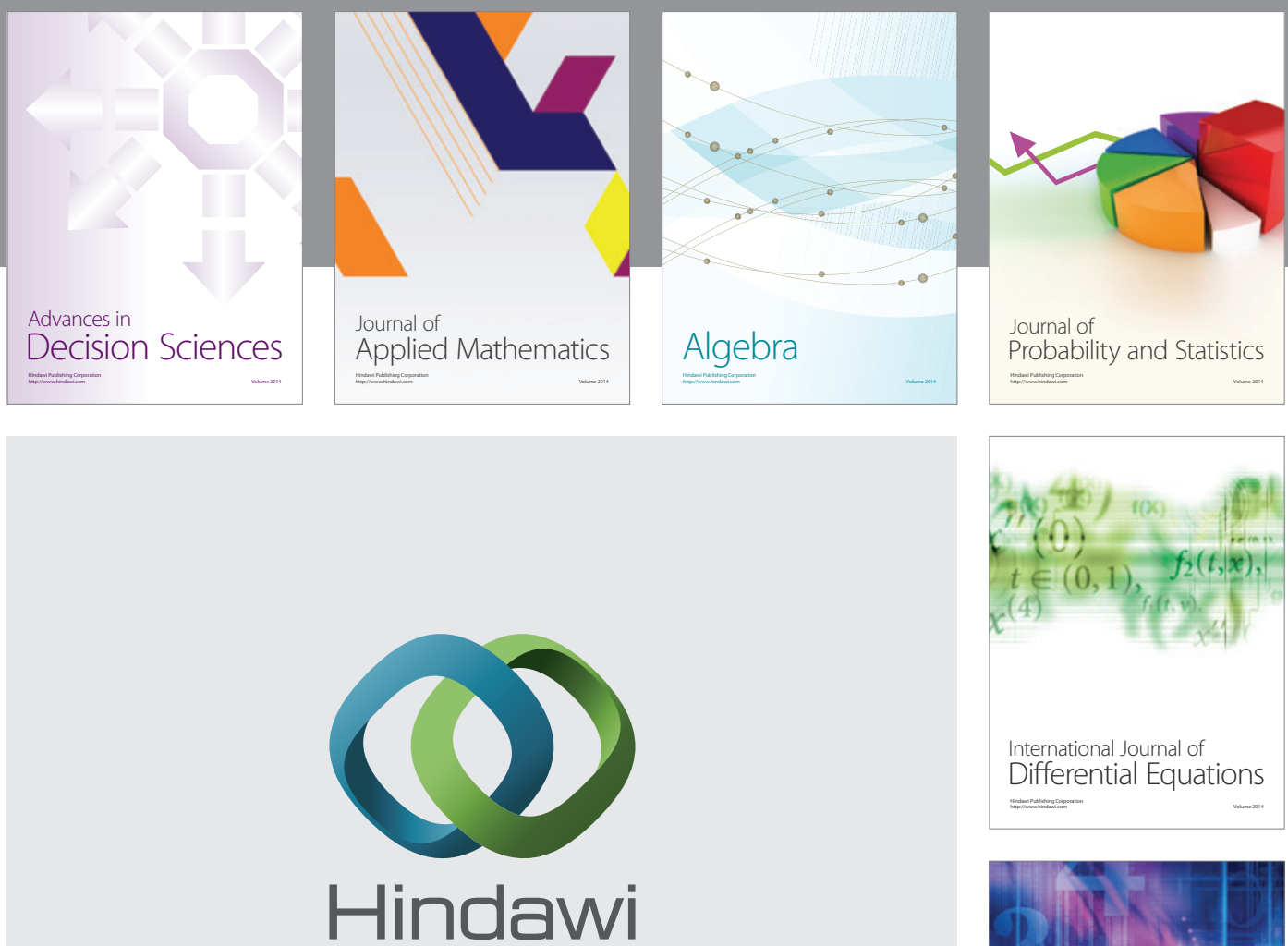

Submit your manuscripts at http://www.hindawi.com
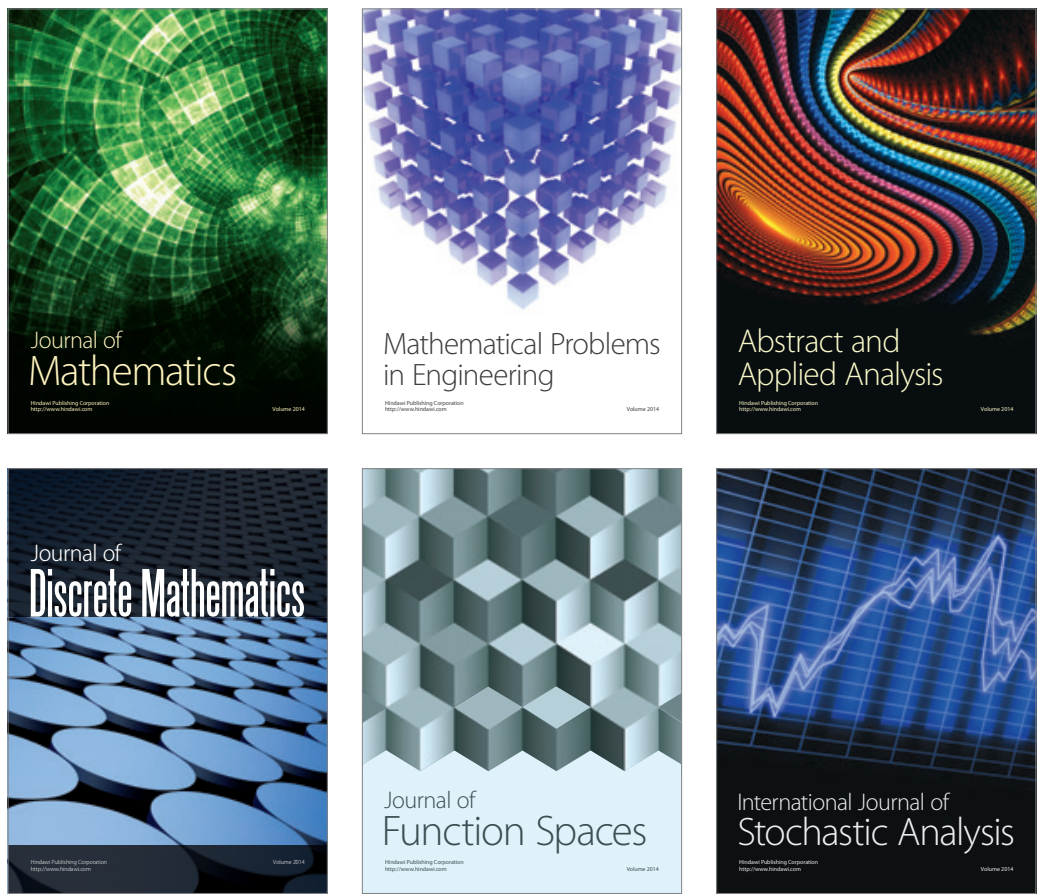

Journal of

Function Spaces

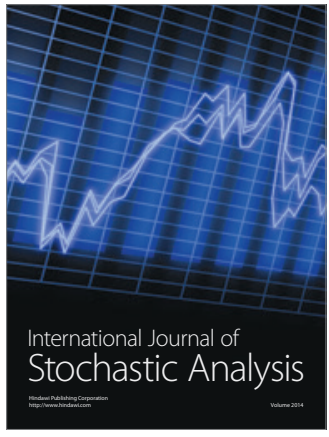

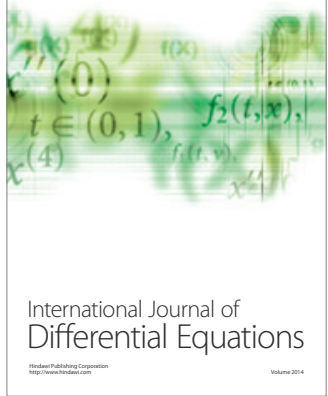
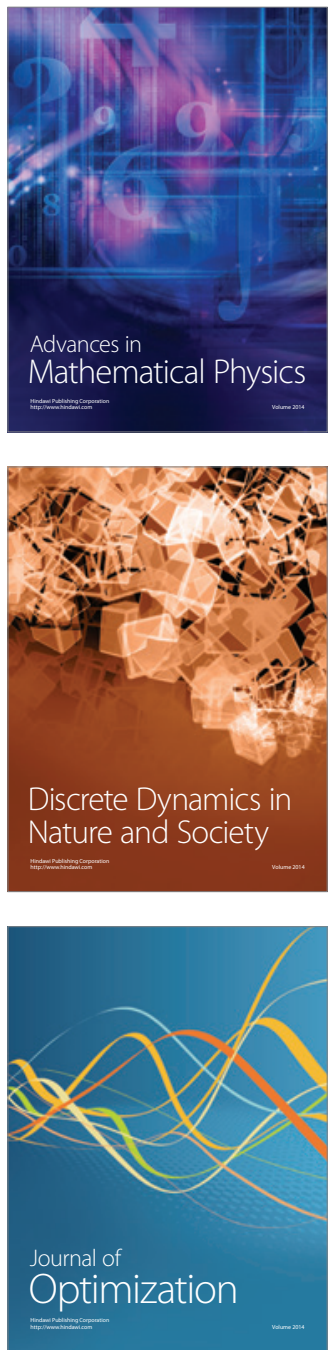